\title{
Web-Based Electronic Data Collection System to Support Electrochemotherapy Clinical Trial
}

\author{
Ivan Pavlović and Damijan Miklavčič
}

\begin{abstract}
Many branches of the healthcare industry are being influenced by information and communication technology (ICT). Clinical trials are not an exception. Despite this fact, more than $\mathbf{7 5 \%}$ of clinical trials data are being collected on paper records. Recent ICT advances, such as broad acceptance of Internet Technology which are rapidly improving electronic data collection (EDC) tools, however, may soon reduce this percentage of "paper" supported clinical trials. In this paper, we present our Web-based EDC system designed to support a small-scale research-oriented clinical trial for establishing standard operating procedures (SOP) for electrochemotherapy with a new medical device, named Cliniporator. The definition of the SOP can only be based on a comprehensive analysis of collected data and results of clinical trial. Therefore, it is necessary to record treatment efficiency and, in this respect, to carefully follow and collect treatment parameters. We thus established central database and the Web application for filling database with data submitted by users from distant medical centers across Europe. Also, we enabled transmitting of data stored on the local Cliniporator medical devices to the central database as well as submitting of tumor images and marking of tumor nodules on interactive human map developed in Macromedia Flash. We provided users with dynamically generated basic statistics, and, several times during data collection process, we performed statistical data analysis. In order to assure high quality of data in a database, we included several mechanisms: automatic data validation, digital signatures, the form completeness notification system, e-mail alerting of completed forms, and "check tables." After 13 months of using the systems, we performed a simple usability evaluation of the system by asking users to answer to a questionnaire, and here we present the results. With this paper, we try to share our experience and encourage others to exploit Internet and Web technologies to improve clinical trials data collection, follow up, and data analysis.
\end{abstract}

Index Terms-Clinical trial, computer applications, electronic data collection, Internet.

\section{INTRODUCTION}

$\mathbf{S}$ OCIETY is continuously being influenced by modern information and communication technology (ICT). Each and every economic branch is somehow affected by the use of ICT. Healthcare has already profited extensively from these developments [1]. With the term "healthcare" we describe the industry associated with the provision of medical care to individuals. Probably one of the strongest breakthroughs of ICT in the healthcare was introduction of electronic patient records (EPR). This is

Manuscript received April 26, 2005; revised February 21, 2006 and March 30,2006 . This work was supported in part by the ESOPE (QLK3-02002-2003) project funded by the European Commission and in part by the Slovenian Research Agency.

The authors are with the Faculty of Electrical Engineering, University of Ljubljana, SI-1000 Ljubljana, Slovenia (e-mail: ivan@lbk.fe.uni-lj.si; damijan@svarun.fe.uni-lj.si).

Digital Object Identifier 10.1109/TITB.2006.879581 the main reason for the increase of resources that the healthcare industry allocates for the ICT from $2 \%$ of its revenues during the 1990 s to $5 \%-7 \%$ in recent years [2].

EPRs bring significant improvements in collecting and processing clinical trials data. However, data stored in EPR are not suitable or do not include all the required data for the purpose of clinical trial. Therefore, collection of clinical-trial data usually requires specific data-collection software. Several vendors offer electronic data collection (EDC) tools to support clinical trials. Even more, they are expanding their EDC offers into broader eClinical suites [3]. Those involve: EDC, clinical-data-management systems (CDMS), drug-safety monitoring, trial management, site management, vocabulary management, eSubmission, electronic patient diaries, interactive voiceresponse systems, and investigator management.

Despite the fact that EDC tools have been available for more than two decades, clinical trials are still mainly conducted using paper data collection as the primary tool (over 75\% [4]). The reason for this can be partially ascribed to the fact that standards for exchange of clinical data among different parties still have to be developed, and that these standards should be extended to facilitate data collection at the investigation site. Present technological applications often do not have adequate functionality to meet current needs. In addition, available commercial applications are usually economically acceptable only for large-scale trials. And, even then, they can hardly meet specific trial needs, like integration of other data sources and data from medical devices. It is inherent to the trial process that it can be modified during the study, and thus EDC system should be flexible to support these modifications. With respect to all the requirements listed above, it is still hardly possible to expect a single system that could meet the variety of different trial needs.

In this paper, we present the EDC system, which we developed in support of multicenter clinical trial performed in the frame of European Commission 5th framework demonstration project European Standard Operating Procedures of Electrochemotherapy and Electrogenetherapy (ESOPE). Following are the starting points for developing our EDC system:

- two years of clinical trial using new medical device;

- trial is running on different locations (medical centers) scattered around Europe;

- allowing collection of data and follow-up of progress in the project;

- intermediate and final analysis of collected data for a purpose of defining and validating standard operating procedures for electrochemotherapy;

- maintenance during the project and at least for additional two years (preferably at no cost). 
Easy deploying, maintenance, and upgrading of the EDC system, followup of the progress in the project, and scalability of the system were set as our major goals to be achieved. As per these goals and starting points, following is our strategy for developing our EDC system:

- centralized database;

- development of an application for easy data submission to the central database;

- computer- and platform-independent solution;

- remote-application maintenance and upgrading;

- application must work or be accessible inside the clinical network;

- respecting the "privacy" and ownership of each medical center's data.

The system we built is based on Internet technologies to allow for mobility, to enable multimedia content, and to achieve wide user acceptance. We participated in the analysis of collected clinical data, and here we present the complexity of that process. After 13 months, we performed a basic-usability evaluation of the system, which was done through the Web-questionnaire filled by the users, and in this paper, we present a summary of their responses. Additionally, we built another Web application to enable supervision of device performance and disposable consumables use for the industrial partner supplying medical centers with the medical devices used during the study.

\section{ESOPE PROJECT}

Recently, as part of the Cliniporator project funded by the European Commission (QLK-1999-00484), a new medical device, called Cliniporator, was developed. It was specifically designed for clinical electrochemotherapy and electrogenetherapy. This device was designed to deliver trains of short high-voltage pulses and longer low-voltage pulses in order to electroporate the tissue and enhance drug including DNA delivery into cells. During the pulse application, the control unit measures voltage and current, which allows monitoring and immediate control of the treatment performed, if necessary. After the pulse application, voltage and current measurements are stored in the local database on the device.

After the Cliniporator project, the medical device Cliniporator was in the stage of testing in clinical environment. The testing was performed in four medical centers in Europe within the ESOPE project (QLK3-02002-2003) funded by the European Commission. The aim of the project was to define and validate standard operating procedures (SOPs) for electrochemotherapy and electrogenetherapy. The definition and validation of the SOP is based on comprehensive intermediate and final analysis of collected data and results of clinical trial. It was, therefore, necessary to record treatment efficiency and, in this respect, to carefully follow and collect treatment parameters including the drug used, anesthesia and sedation, pulse applications, tumor type and location, and response to the treatment.

For collection of data acquired in electrochemotherapy clinical trial, standard paper forms (case report forms-CRFs) were prepared. The CRF consists of a number of subforms, the extent of which depends on the number of treated tumors and the num- ber of sessions required to treat each tumor. The CRF includes patient's general data, his/her medical history, tumor-treatment data, and tumor response. Tumor treatment can be repeated, if necessary. For each patient, medical personnel have to fill in approximately 40 pages of forms, on average.

The paper-based forms were intended to be used for documentation purposes, and for clinical-data analysis. We established a unified database of all collected data in medical centers involved in the study. This central database is intended to offer a better overview of trial data and easier follow up of the progress on the project, and to serve as a source for comprehensive analysis of collected data and results during and also after the end of the project.

The central database was planned to be daily filled with data from different locations around Europe. For this purpose and to fulfill expectations, we built a Web-based EDC application (Cliniporator Web-Recorder) that provides a user-friendly interface for remote filling of central database, so the medical personnel can easily carry it out. Each user from the medical centers involved in the study is able to submit his/her patientand treatment-related data directly to the central database.

Due to the distances between the centers, the EDC application has to be easy to deploy, maintain, and debug. These were the reasons to develop a Web-application. EDC application has to provide two main services:

- digital CRF-digital forms for submitting CRF data to the central database;

- local database upload-a module for transferring data from the local Cliniporator-device databases to the central database.

These two services are the core of our EDC system. The schema of the system is presented in Fig. 1. However, the advantage of the proposed Web-based solution is that any other service can easily be added, which we also demonstrated. During the trial process, we extended the initial application by adding two additional services. One was the statistic service that offers dynamically generated statistics charts for already submitted data, which allowed the coordinator to follow the progress of the project. The other one was added to be used by the device manufacturer for tracking the device functioning and electrode supplies and is fully described in [18].

\section{Central Database}

The central database is a relational database established on the Microsoft SQL Server 2000. At this moment (after 32 months of continuous use) its size is $1.5 \mathrm{~GB}$ (including more then 1800 tumor images). It consists of more than 30 tables. Most of the tables are related to the CRF data, but there are also tables dedicated to storing images, data uploaded from local databases and users' data, and permissions and activities.

Following is the trial data stored in the central database:

- patient data (demography, medical history, physical examination, etc.);

- therapy data (sessions, evaluation visits, follow-up visits, etc.); 


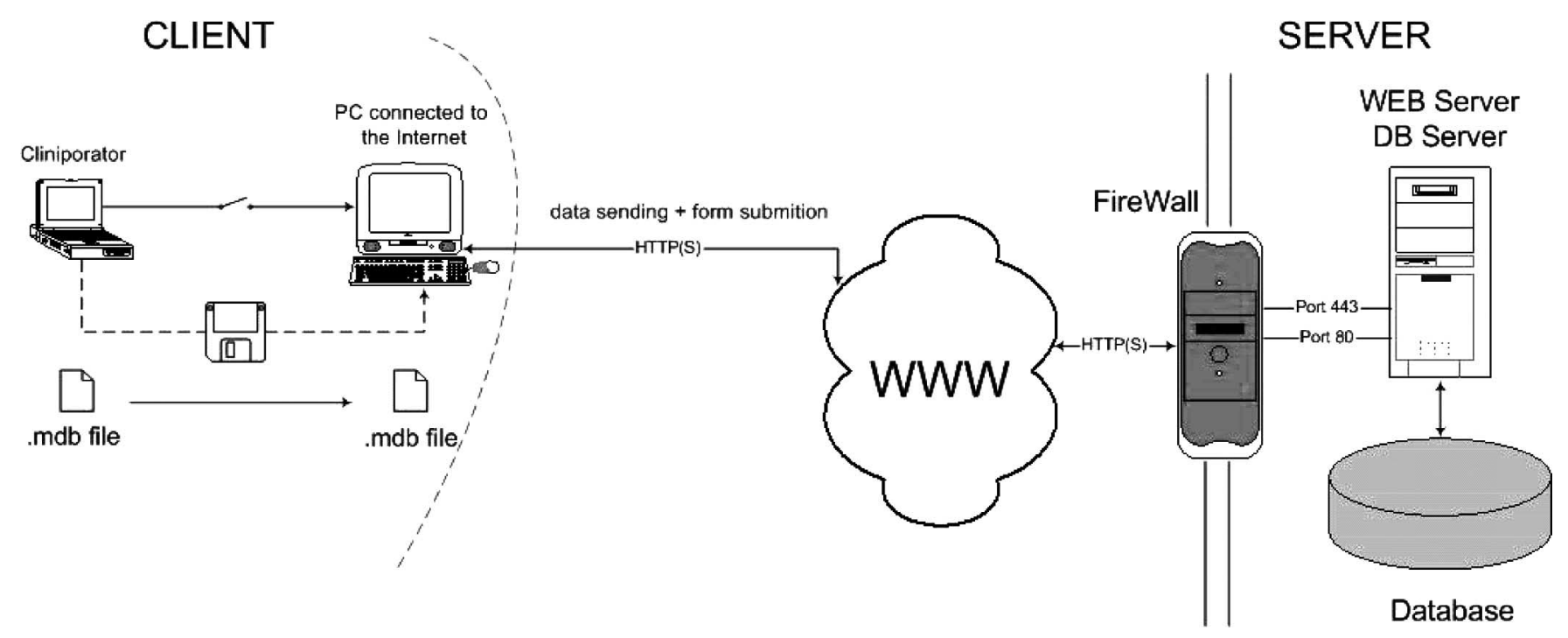

Fig. 1. Schema of the EDC system. On the client side, the user's computer needs just an Internet browser to access the server in order to add and edit the data in the central database. All the processing is performed on the server side, except the data validation, which is performed by the client browser.

- data from the local databases of Cliniporator medical devices;

- digital images of tumor nodules in different phases of therapy and follow up.

Data uploaded from the local Cliniporator devices have to be transferred from the Microsoft Access database to the MS SQL Server 2000 central database. This process is automated through the data transformation service (DTS) package. The DTS package extracts data from the tables in the MDB file which the user uploads to the server, does necessary transformations, and stores transformed data in the corresponding tables in the central database.

In order to prevent a hazardous loss of data, two copies of backup of the database are automatically created once a week: one on the local hard disk, and the other on the tape. In addition, two copies of backup of transaction log are stored once a day. The scheduled jobs executed on the recurring basis are created for these purposes.

\section{WEB-BASED EDC APPLICATION}

The web-based application for electronic data collection, which controls the users, interface to the system, is the core of the system. We named it Cliniporator Web-Recorder. It is a set of active server pages (ASP), which are running on the Microsoft Internet Information Server 6 (IIS 6) Web server. It processes users' requests, queries the database, generates web pages, and runs other processes like storing data from submitted local databases to the central database, storing submitted images, online generating of data export tables or basic statistics, or sending e-mail alerts to the coordinator or other people in charge of following the progress, the control of data entries, etc.

The Cliniporator Web-Recorder currently offers several functionalities:

- web forms (digital CRF) for data entry;

- upload of images;

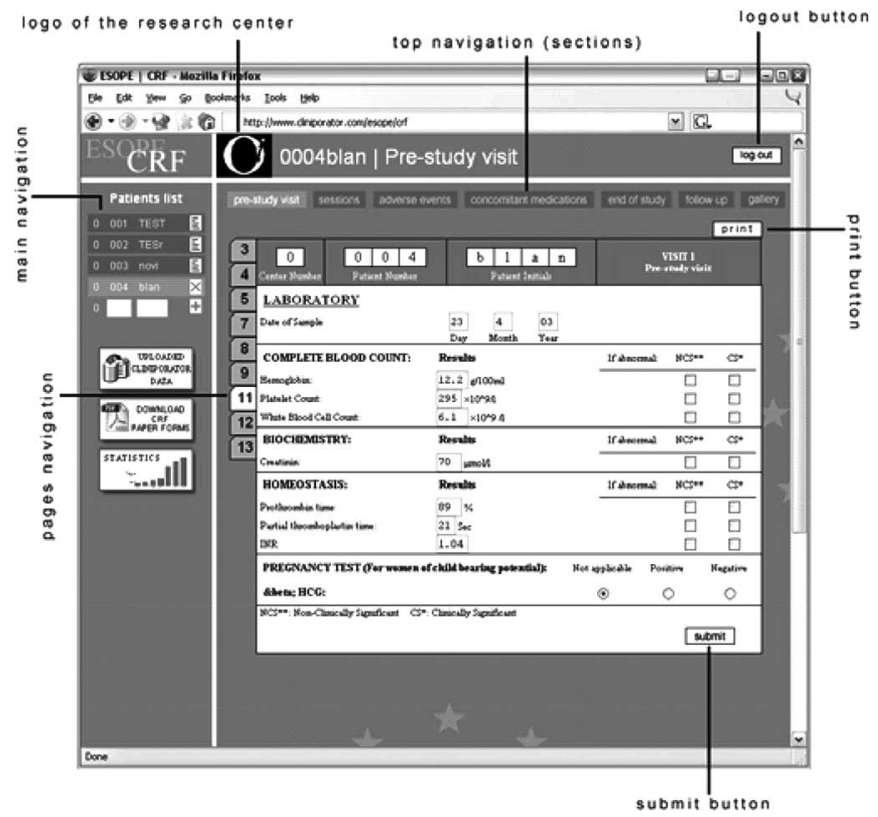

Fig. 2. Electronic CRF page. The users enter the data to the central database through the set of Web forms similar to the paper-based CRF.

- interactive human map for marking tumor locations;

- local MDB database upload from the Cliniporator medical device;

- basic online statistics;

- interactive tumor-history graph.

\section{A. Web-Forms (Digital CRF) for Data Entry}

Web-forms (digital Clinical Report Forms-CRFs) are formlike Web pages (Fig. 2). Through digital CRF, users submit patient and treatment data to the central database. A digital CRF has the same structure and design as the paper-based CRF. Digital CRF are organized in sections (prestudy visit, sessions, 
evaluation visit day 15 and day 30, follow-up visits, end of study, concomitant medications, and adverse events), and some of these sections are further divided into several pages.

Digital CRF contains automatic data validation. This entry checking mechanism warns the user if he/she mistypes or enters meaningless data. This is performed by client-side JavaScript code embedded into the Web pages. As the validation routine is performed on the client computer, there is no need for additional client-server communication, which reduces the Internet traffic and improves the application speed.

At the end of every section, the user has the opportunity to "digitally sign" the section, by filling/entering his/her password in the appropriate signature-input field. By signing a section, the corresponding forms are "locked" and all further modifications are disabled. In this way, the user confirms that the data in the forms are complete and valid. Signing of the section triggers an e-mail alert. The data manager and the project coordinator receive an e-mail, which informs them that the forms are completed, so they can include data in the data analysis. If any mistake in the signed data is detected, a user who signed the data has to send the request to unlock the section to the project coordinator, who approves the changes and forwards the request to the database administrator. The database administrator is the only one who is able to access directly to the central database and edit the data. A copy of the unlock request is kept as part of the project documentation.

We integrated additional mechanism for improving quality of data in the database: the form-completeness notification. This mechanism is there to notify users of yet incomplete forms. Namely, when the user completes any particular section or part of the section, he/she signs it. Automatically all the corresponding links to the pages in the page navigation bar are changed from normally black color to "gold." Therefore, in order to complete the forms, the user has just to follow the black links to the pages that are not yet filled. Similarly, button links to the sections in the section navigation get "gold stamp" if the whole section is signed. And also, if all the forms of the particular patient are signed, the "gold stamp" appears above the edit icon in the patient navigation. This enables users to follow nonstamped links and quickly find incomplete forms and fill them.

\section{B. Upload of Images}

Image upload enables storing of digital tumor images into the central database. Images, captured with digital camera, are uploaded in their original size. During the upload process, a smaller image, suitable for displaying, as well as a thumbnail of the image, are dynamically generated and also stored in the database. All the images are stored in the JPEG format. Users can add a caption and a description to each image. Images can be added at all phases of the treatment (prestudy, sessions, follow up, etc.). In the image gallery, the user can review all the uploaded images of a single patient. Thumbnails of images are grouped by tumor nodules, and sorted by the date of capture. By selecting appropriate thumbnail, the user can see the large image subtitled with submitted comments. The user can also download the originally submitted image. Image gallery proved

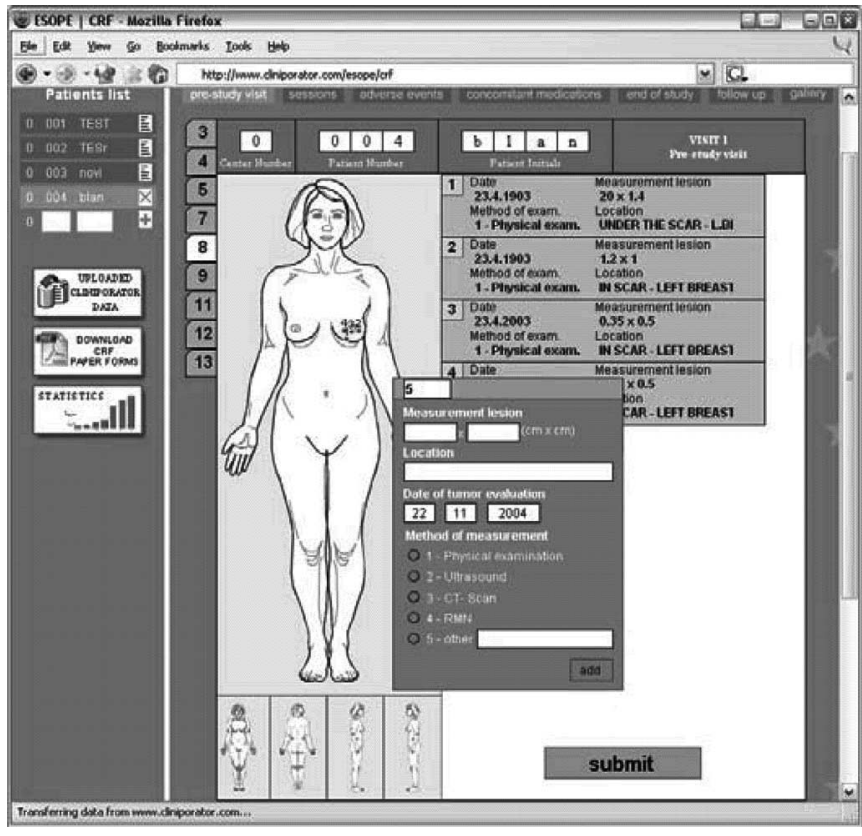

Fig. 3. Interactive human map. Users mark the tumor nodules and enter basic data on the digital human map developed in Macromedia Flash.

to be very useful for visual inspection and observation of tumor changes.

\section{Interactive Human Map for Marking Tumor Locations}

The purpose of the interactive human map is visual presentation of the tumor locations on the human body. According to the patient's sex selected within the CRF, appropriate body map is displayed. Users can switch between four views: front, rear, left, and right. By simply clicking on the map, the user can mark the location of the tumor, and then submit basic data about the tumor (such as location and measurement of lesion) and upload corresponding images. During the sessions, the user can select on the map which of the tumors, identified and recorded during the prestudy visit, are treated. The map was developed in Macromedia Flash Player 6, and it exchanges XML-structured data with the Web server. The interactive human map is shown in Fig. 3.

\section{Local MDB Database Upload From the Cliniporator Medical Device}

Upload of local Cliniporator device databases is also performed through the Internet browser. The user simply selects the local database file, fills in upload comment, and selects the submit button. The rest of the process is automatic: application saves uploaded file on the server; stores upload information (date and time, user ID, name of the file, and comment) in the central database; and then copies data from the uploaded file to the central database. A DTS package, which we developed, takes care of data copying and overwriting. At the end of the upload process, the user is informed of the upload success/failure.

In the list of the uploaded data, the user can check all the data uploaded from his/her center. Data are sorted by patients, 


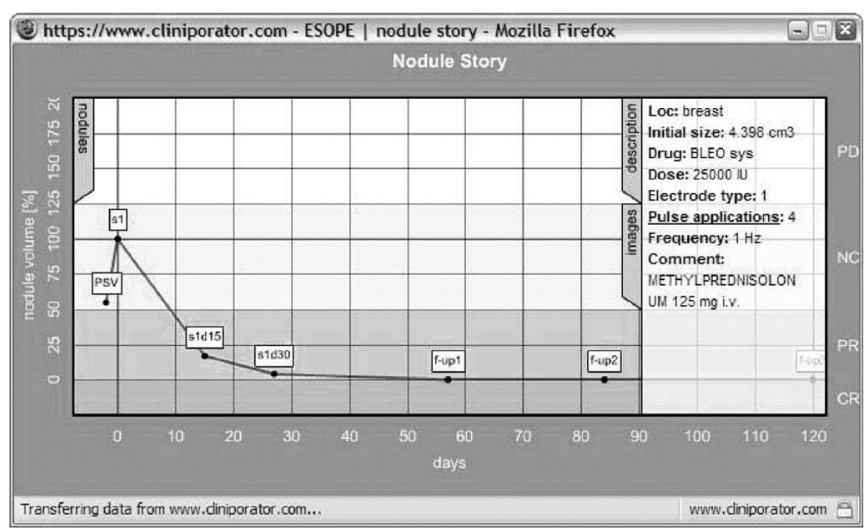

Fig. 4. Interactive tumor-history graph. On the graph, the user can see the timeline of the nodule size and therapy data as well as tumor images and a list of all the patient nodules (not presented here).

sessions, nodules, and pulse applications. It is possible to check data for every particular pulse application. A pop-up window with pulse description contains a table with the pulse data (like device settings and pulse measurements), and a pulse figure dynamically generated from the recorded voltage and current measurements.

\section{E. Basic Online Statistics}

Basic statistics, which allow to follow up the progress on the project, are dynamically generated from the data in the central database. Information about the number of treated patients per center as well as the number of ended therapies, number of tumor treatments and uploaded corresponding local databases, and distribution of applications of different electrode types and different drugs used is available online. All centers have access to these statistics and can compare their activities with others. Some of the statistics (usually local statistics) can be dedicated to a particular center and, therefore, hidden to others. Statistics are presented in Macromedia Flash 3-D charts.

\section{F. Interactive Tumor-History Graph}

Users have access to the tumor history for each of the patients submitted from the same center. Tumor history is a record of changes of tumor-nodule sizes through the evaluation and follow-up period after treatment. We designed interactive Flash presentation of the tumor history for each patient in the database (Fig. 4). This presentation contains the list of the tumor nodules for a particular patient and graph with timeline of nodule size for each of the nodules. Users can show or hide any of the timelines to clear the graph. Each timeline is accompanied by data of tumor initial size and location, therapy, drug, and pulse parameters, as well as tumor images. The interactive tumorhistory graph is dynamically generated from the data stored in the database on demand.

\section{SECURITy MEAsures}

The data security is assured on three levels: server level; data transmission level; and application level.
TABLE I

DATABASE ACCESS PERMissions

\begin{tabular}{|c|c|c|c|c|}
\hline & User & $\begin{array}{l}\text { Read-Only } \\
\text { User }\end{array}$ & $\begin{array}{l}\text { Guest } \\
\text { User }\end{array}$ & $\begin{array}{c}\text { Device } \\
\text { Manufacturer }\end{array}$ \\
\hline $\begin{array}{l}\text { Cliniporator Web-Recorder } \\
\text { CRF }\end{array}$ & $r+w$ & $\mathrm{R}$ & $r+w$ & $X$ \\
\hline $\begin{array}{l}\text { Cliniporator Web-Recorder } \\
\text { Basic Statistics }\end{array}$ & $\mathrm{R}$ & $\mathrm{R}$ & $\mathrm{R}$ & $X$ \\
\hline $\begin{array}{l}\text { Cliniporator Web-Recorder } \\
\text { Data Extraction Tables }\end{array}$ & $r$ & $\mathrm{R}$ & $r$ & $X$ \\
\hline $\begin{array}{l}\text { Cliniporator Service Module } \\
\text { Data Extraction Tables }\end{array}$ & $X$ & $\mathrm{X}$ & $\mathrm{X}$ & $\mathrm{R}$ \\
\hline $\begin{array}{l}\text { Cliniporator Service Module } \\
\text { Querying the Database }\end{array}$ & X & $X$ & $X$ & $\mathrm{R}$ \\
\hline
\end{tabular}

Access to the database is granted differently for different user types and can be assigned according to the needs and agreements. $(r-$ read data from the same center; $\mathrm{w}$ - edit data from the same center; $\mathrm{R}$ - read data from all centers; $\mathrm{X}-$ no access)

On the server level we have two main security measures: the firewall and the database backup. The computer, which runs the web server and the database server, is set behind the firewall. The firewall examines all the data traffic coming in and out of the server computer and blocks the traffic which does not meet the specified security criteria. The data stored in the central database are frequently backed up according to the policy described earlier, in the "Central database" section.

On the data-transmission level, all the communication between the client and the server is encrypted through the secure socket layer (SSL) protocol. SSL is an encryption protocol that encodes data sent over the World Wide Web, rendering it unreadable to anyone intercepting the transmission. When an SSL session is started, the server sends its public key to the user's browser, which the browser uses to send a randomly-generated private key back to the server in order to have a secret key exchange for that session. Developed by Netscape Communications Corporation and RSA Data Security, Inc., SSL is the leading security protocol used on the Web.

On the application level, we perform authentication and authorization of users. The client authenticates him/herself by username and password. The application checks the user identity in the central database where the table of users and their permissions are stored. Based on the user's identity, authorization allows appropriate access to the resources (e.g., it enables access only to data uploaded by his/her medical center-see also Table I). However, the coordinator has the right to review the data of all the centers. Contrastingly, the industrial partner has access to limited subset of data, of all medical centers, that are related to Cliniporator device performance and electrode usage. Accessing whole data or specific subset depends on the agreement between parties involved and can easily be granted and changed, if necessary. For the moment, data-access permissions are granted as presented in Table I.

\section{DATA QUALITY AND DATA ANALYSIS}

The main aim of the ESOPE project, and thus also the main purpose of the Cliniporator Web-Recorder, was to aid 
in establishing SOPs for electrochemotherapy and electrogenetherapy with the Cliniporator device. This aim requires comprehensive analysis of the collected data at different times during the clinical trial.

Analysis of the medical-trial data usually may not be done automatically, especially when the trial is running on different locations and performed by different personnel. The human interaction in this process is absolutely necessary. An expert can easily interpret different artifacts and, if necessary, exclude some data from the analysis. Direct statistical analysis of the data in the database can lead to erroneous conclusions. It is too optimistic to expect that all the data are entered correctly and all the trial is performed perfectly. However, data analysis is necessary and useful. It shows correlations between different treatment parameters, and proves or denies our hypotheses, leading to better understanding of the biological processes.

Data quality control appeared to be very demanding and essential. In order to improve data quality source verification we created "check tables" - on the fly-generated tables of data extracted from the central database, which were available to all the users through the Cliniporator Web-Recorder. These tables contained only the data related to the several data analyses essential for the ESOPE project. In these tables, users were more easily checking for missing data or unusual values and then correcting these data in the Web forms. The coordinator also had access to "check tables" and he was assisting in the correction process.

We enabled form-completeness-notification system and e-mail alerting of completed forms. In this way, we tried to move most of the data controlling process from coordinator to users themselves, and to shift control of form completeness and data quality control from serial to parallel process. With this solution, data review can begin as the soon as data have been entered and the quality of data in the central database can be high even during the data entry, without locking the database. This high confidence of data in the database during the study enables frequent data analysis and, thus, better directing of the study.

In the central database, data are organized in numerous tables. But, for the statistical analysis, the most suitable form of data representation is a "flat table" (one single table with all the required data included in it). Therefore, we usually have to extract data from a set of tables in central database and to organize them in a flat table. This extraction of data requires complex queries on a set of tables. However, once derived, extraction can be automatic for repetitive analysis. The result, i.e., the "flat table," serves as a source for the data analysis, graphical presentation, and statistical analysis. Graphical presentation sometimes requires further transformations and reorganization of data in the result table. Also, some data analysis and statistical evaluation, specially the ones which are performed frequently and/or regularly, can be automated and repeated as the number of patients included is increasing.

\section{USABILITY EVALUATION}

As the human-computer interaction is increasing its role in everyday processes, the importance of usability engineering [5] has become obvious. This resulted in development of new methodologies for usability studies and improvement of the existing ones [7]-[13]. However, performing a complex usability study can be a very expensive process, and, therefore, not always applicable. However, some authors [14], [15] claim that gathering feedback information from four to five users of the system is enough to discover $80 \%$ of the usability problems of the system. We decided to perform a basic usability evaluation with a simple structured questionnaire, with having in mind all the weaknesses of this approach [16]. In this way, we believe that we could find some of the features of the system that were necessary to improve in order to reach better usability of the system. In addition, through constant interaction in meetings and direct contacts with users and their feedback we were discovering advantages and drawbacks of the system. In this way, we believe that we could find some of the features of the system that were necessary to improve in order to reach better usability.

After 13 months of running the system, we designed a structured questionnaire to obtain users' opinions about different aspects of the interface and their use of it. Following the usability evaluation done by Chadwick et al. [17], we divided the 46 questions under six heads: 1) attitudes toward paper-based system; 2) perceived benefits of electronic records; 3) user satisfaction and ease of use of electronic interface; 4) problems when using the system; 5) likely future usage; and 6) attitude toward data security.

The questionnaire was Web based and available to each user of the system. As there was only one registered user per each medical center involved in the study, we received four completed questionnaires. Even though the number of users and responses is low, most of the obtained answers clearly pointed to some of the advantages and disadvantages of the EDC system. The summary of the results of the questionnaire is presented below. (The complete results of the questionnaire are presented in [18].)

\section{A. Attitudes Toward Paper-Based System}

Most of the users were satisfied with the paper CRF. They did not find it too complex, but some of them expressed to have difficulties in searching for data in paper records. Moreover, all of them agreed that the analysis of data collected in the paper forms is a demanding process.

\section{B. Perceived Benefits of Electronic Records}

Users expressed great understanding of benefits that the electronic records bring. They all shared the opinion that the system like this brings optimization of the overall process, and that it improves the analysis of collected data. They mostly had a positive attitude toward complete substitution of paper records with electronic records.

\section{User Satisfaction and Ease of Use of Electronic Interface}

This portion of questions was the most important for the evaluation of the Cliniporator Web-Recorder application. We expected here, on one side, to see the flaws in solutions we built, and on the other side to get a confirmation for our ideas that we integrated into the system. 
The overall evaluation of the application was positive. Users found the application fast enough for their needs. They were completely satisfied with the interface design and navigation concept. In their opinion, entering and searching for information was simple. The idea of having Web forms similar to the paper forms and organized into many short pages instead of few long-scrolling pages was well accepted. Users considered the interactive human map extremely useful. The automatic check of filled data was useful, as well as the signing of the forms.

Other application features, like image gallery, upload of the local-database data, and presentation of dynamic statistics were also positively accepted. The users considered useful both storing the images into the central database and accessing them in the image gallery. The upload of local-database data was convenient, and the overview of uploaded data useful. In their opinion, beside the electric-pulse data, the presentation of the electricpulse figure was also important. Also, they were positive of putting basic statistics on the Web.

Apart from one user, all others had positive impression of the speed of fixing bugs in the system. However, they all agreed that the process of upgrading the system was convenient and not disturbing their work.

\section{Problems When Using the System}

In this portion, we tried to identify the weakest points in the system. As expected, filling of both paper and electronic CRF was inconvenient practice. This is something that will probably stay for considerable time, and all we can do is to make these two processes as similar as possible.

Our concern that the paper forms are more suitable for use during the medical procedure was confirmed. This is something that is mostly a question of technology advancement, and it is probably just a matter of time when this problem will be overcome. For example, use of tablet personal computer (PC) or pocket PC with wireless local area network (WLAN) connection would enable filling of electronic records even during the medical procedure. This procedure, however, needs to become acceptable for patients and medical doctors.

The three most important questions in this portion, from our point of view, were pointing the system accessibility and reliability. The users appraised the system as reliable, which is good. All of them, except one user, found the login procedure convenient. The main problem in the system was connectivity, as two users emphasized that it was happening occasionally that they could not access the system. We believe that the reason for this lies in significant system improvements that we introduced (establishing of secure connection and migration to a new server operating system) a few weeks before asking users to fill out the questionnaire, which coincided with the time of intensive data-verification activities. Unfortunately, this caused frequent system restarting in the days of the highest user activity. This, however, could be avoided by proper planning of activities and their harmonization with users' activities.

\section{E. Likely Future Usage}

The aim of this portion was to evaluate the present use and to highlight the direction of future improvements. The outlook for likely future use is good with each of the users stating that they would like to use the system even after the end of the project. The users estimated the overall system usefulness as positive, and expressed the wish to use similar EDC systems for other clinical trials. They did not agree on the advantages of Web-based medical-record system over the usual (non-Web based) computer medical-record system. Nevertheless, all the users agreed that an obvious advantage of this system was the support of multimedia content. One of the users was, however, concerned over the use of medical applications over the Internet.

Despite good evaluation results of system performances, users pointed out that most of the performances should be enhanced. They mostly expected that the application speed and reliability should be improved. One of the users clearly expressed that the application design should be improved. Another wished that the navigation be improved. Still another expected more dynamic statistics to be introduced.

\section{F. Attitudes Toward Data Security}

The last portion of the questionnaire referred to the system security. The general attitude of users was that the security measures were important in medical applications on the Web. They were willing to accept decrease in the system performances with an improvement in the overall system security. And finally, users expressed trust in the experts who implemented the system and thus showed no concern for the implemented security measures (log in with password and encrypted connection).

\section{DISCUSSION}

Data presented by Paul et al. [19] show that in the last ten years, there has been a noticeable increase in the number of reported online clinical trials. This increase is driven by improvements in computer technologies with a special contribution of Internet technologies, which enabled simple solutions for many trial management and communication problems. Since the introduction of the Internet-based solutions for electronic data collection in medical applications, many of them were offering partial or complex solutions for clinical trials. In the beginning, authors were focused on developing generic systems that could enable quick and generic development of medical forms [20], [21]. Some authors were focused on different technological aspects and solutions like Java [21], XML [22], security aspects [23]-[25], or data validation [26]. However, according to our review of numerous available international journals, there are very few articles about noncommercial systems to support multicenter trials that integrate electronic data collection, data management including data validation, trial management, data reports (online statistical analysis), and strong security measures (including authentication, authorization, data encryption through SSL, and servers' protection) [27]-[32]. However, Brown gives an overview of the EDC market and commercial vendors offering software for clinical trials [3]. 
Based on the Internet technologies, our EDC system enables efficient collection of clinical-trial data within the ESOPE project from distant medical centers, thus easing and empowering trial management and result analysis. It enables data storage and backups, as well as control of entries, timeliness, and completeness of the data. In our opinion, the key feature of every EDC system is assuring high data quality. There are several approaches in achieving high data quality including: real-time data validation, automated e-mail notifications [32], presentation of complete medical-text summary [26], [31], different types of users with different roles in the process (e.g., user, investigator, monitor, and administrator) [28], and digital/electronic signatures [26], [28]. We tried to surpass this usually costly process by empowering user's autocorrection of submitted data through the use of "check tables" and completeness notification system, and by enabling parallel submission and monitoring of entries. We enabled different data-access permissions for different user types in order to support different roles that participants have in the trial.

This system was not only easy to implement but was also easy to maintain, which were among the initial goals. The system is scalable and flexible as it allows adding new functionalities and changing the existing ones. In two years of running the EDC system, we enlarged the initial set of functionalities with generation of dynamic basic statistics, image gallery, pulse-description window, and few smaller improvements for automatic data validation. Recently we added two important features: e-mail alert of submitting concluded form, and form-completeness-status notification.

The described EDC system can be easily expanded with new interfaces. We have already added a Cliniporator Service Module interface, which enables manufacturers of the Cliniporator device to query the central database for data essential to device functioning and electrode supplies [18].

The EDC system that we developed does not require advanced computer knowledge on the part of the medical personnel who use it. It supports multimedia content and interactivity. The implementation of Macromedia Flash animations for medical applications has been extremely well received by users. This along with the image-gallery feature and integration of data from different sources (data entered through the Web-forms and Cliniporator device local databases) makes our system one of a few noncommercial EDC systems incorporating these features.

Our EDC system includes security measures necessary for medical applications. Finally, as assessed by the questionnaire, our EDC system is well appraised and accepted by users themselves. The users showed their interest to continue using the system even after the end of the project. They also confirmed their need for similar systems for other clinical trials.

During two years of trial and system operation, the system was stable and reliable. Users were filling medical forms, submitting images, and sending local-database data. It successfully passed challenges like migrating server side of the system to the new operating system (from Windows Server 2000 to Windows Server 2003) and upgrading security measures by introducing security socket layer. A lot of smaller modifications were in- stantly performed. At users' requests, few new features were added without disturbing their normal work.

\section{CONCLUSION}

In this paper, we presented a custom Web-based EDC system that we developed to support ESOPE clinical trials and also the usability evaluation of the system done through the questionnaire answered by users after using the system for 13 months. This EDC system had to enable data collection and frequent intermediate data analysis during at least two years of multicenter clinical trial.

Based on our experience, Web-based applications are suitable for distributed medical instrumentation service and monitoring. Another advantage of using Internet technologies is the support of multimedia content. Easy implementation of image, sound, video, or interactive animation content brings many possibilities for different practical solutions. Our image acquisition, interactive human map, and tumor-history graph are just some of them.

The Cliniporator Web-Recorder addresses an important challenge to healthcare: how to exploit Internet and Web technologies to improve clinical trials. The nature of the research process is such that it does not stand constraints that commercial EDC software and fixing of trial procedure brings. This is why we believe that many of the trials need dedicated EDC systems, extended with different features useful for the particular study. The Internet technology fills the gap between application development and deployment. This shifts up a gear in research process. Users/researchers can promptly shape the application design and functionalities. The usual two-way process between users/researchers and system developers can bring the maximum from otherwise usually rigid EDC systems. We want to share our experience and encourage others to go on this path.

\section{REFERENCES}

[1] S. N. Laxminarayan, "Editorial: Information technology in biomedicine: Maturational insights," IEEE Trans. Inf. Technol. Biomed., vol. 6, no. 1, pp. 1-7, Mar. 2002.

[2] K. Siau, "Health care informatics," IEEE Trans. Inf. Technol. Biomed., vol. 7, no. 1, pp. 1-7, Mar. 2003.

[3] E. G. Brown. (2004, Sep). Clinical trials' EDC endgame. Forrester Research, Inc. [Online]. Available http://www.forrester.com/go? docid=33344

[4] L. Alschuler, L. Bain, and R. D. Kush. (2004, Mar.). Improving data collection for patient care and clinical trials. Health Level 7 and Clinical Data Interchange Standards Consortium. [Online]. Available: http://nextwave.sciencemag.org/cgi/content/full/2004/03/24/8

[5] J. Nielsen, Usability Engineering. New York: Academic, 1993.

[6] X. Ferré, N. Juristo, H. Windl, and L. Constantine, "Usability basics for software developers," IEEE Softw., vol. 18, no. 1, pp. 22-29, Jan./Feb. 2001

[7] A. W. Kushniruk and V. L. Patel, "Cognitive and usability engineering methods for the evaluation of clinical information systems," J. Biomed. Inf., vol. 37, pp. 56-76, 2004.

[8] C. M. Johnson, T. R. Johnson, and J. Zhang, "A user-centered framework for redesigning health care interfaces," J. Biomed. Inf., vol. 38, pp. 75-87, 2005.

[9] K. Schriver, "Evaluating text quality: The continuum from text-focused to reader-focused methods," IEEE Trans. Prof. Commun., vol. 32, no. 4, pp. 238-255, Dec. 1989.

[10] M. C. Beuscart-Zephir, F. Anceaux, H. Menu, S. Guerlinger, L. Watbled, and F. Evrard, "User-centered, multidimensional assessment method of clinical information systems: A case study in anesthesiology," Int. J. Med. Inf., vol. 74, pp. 179-189, 2005. 
[11] A. Lecerof and F. Paternó, "Automatic support for usability evaluation," IEEE Trans. Softw. Eng., vol. 24, no. 10, pp. 863-888, Oct. 1998.

[12] A. W. Kushniruk, C. Patel, V. L. Patel, and J. L. Cimino, “Televaluation' of clinical information systems: An integrative approach to assessing Webbased systems," Int. J. Med. Inf., vol. 61, pp. 45-70, 2001.

[13] L. V. Vaes, "Thinking aloud as a method for testing the usability of websites: The influence of task variation on the evaluation of hypertext," IEEE Trans. Prof. Commun., vol. 43, no. 3, pp. 279-291, Sep. 2000.

[14] J. Nielsen. (2000, Mar. 19), "Why you only need to test with 5 users," Alertbox. [Online]. Available: http://www.useit.com/alertbox/20000319.html

[15] R. Virzi, "Refining the test phase of usability evaluation: How many subjects is enough?," Hum. Factors, vol. 34, pp. 457-468, 1992.

[16] A. Woolrych and G. Cockton, "Why and when five test users aren't enough," in Proc. IHM-HCI 2001, Lille, France, Sep. 10-14, 2001,vol. 2 pp. 105-108.

[17] D. W. Chadwick, D. Mundy, and J. New, "Experiences of using a PKI to access a hospital information system by high street opticians," Comput. Commun., vol.26, pp. 1893-1903, Oct. 2003.

[18] I. Pavlović, "Web-based medical record system for clinical trials" M.S thesis, Faculty Electr. Eng., Univ. Ljubljana, Ljubljana, Slovenia, 2004.

[19] J. Paul, R. Seib, and T. Prescott, "The Internet and clinical trials: background, online resources, examples and issues," J. Med. Internet Res. [Online] 2005. 7, no. 1. e5 Available http://www.jmir.org/2005/1/e5/

[20] T. Kiuchi and S. Kaihara, "Automated generation of a World Wide Webbased data entry and check program for medical applications," Comput. Methods Programs Biomed., vol. 52, no. 2, pp. 129-138, Feb. 1997.

[21] H. Sippel and C. Ohmann, "A web-based collection system for clinical studies using Java," Med. Inf., vol. 23, no. 3, pp. 223-229, 1998.

[22] P. Wübbelt, G. Fernandez, and J. Heymer, "Clinical trial management and remote data entry on the Internet based on XML case report forms," Stud. Health Technol. Inf., vol. 77, pp. 333-337, 2000.

[23] S. Gritzalis, J. Iliadis, D. Gritzalis, D. Spinellis, and S. Katsikas, "Developing secure Web-based medical applications," Med. Inf., vol. 24, no. 1, pp. 75-90, 1999.

[24] H. M. Chao, C. M. Hsu, and S. G. Miaou, "A data-hiding technique with authentication, integration, and confidentiality for electronic patient records," IEEE Trans. Inf. Technol. Biomed., vol. 6, no. 1, pp. 46-53, Mar. 2002.

[25] M. van der Haak, A. C. Wolf, R. Bradner, P. Drings, M. Wannenmacher, and T. Wetter, "Data security and protection in cross-institutional electronic patient records," Int. J. Med. Inf., vol. 70, pp. 117-130, 2003.

[26] R. G. Marks, "Validating electronic source data in clinical trials," Controlled Clin. Trials, vol. 25, pp. 437-446, 2004

[27] M. Formica, K. Kabbara, R. Clark, and T. McAlindon, "Can clinical trials requiring frequent participant contact be conducted over the Internet? Results from an online randomized controlled trial evaluating a topical ointment for herpes labialis," J. Med. Internet Res., vol. 6, no. 1, 2004

[28] C. D. Lallas, G. M. Preminger, M. S. Pearle, R. J. Leveillee, J. E. Lingeman, and J. P. Schwope, "Internet based multi-institutional clinical research: A Convenient and secure option," J. Urol., vol. 171, no. 5, pp. 1880-1885, May 2004

[29] C. H. Elsner et al., "Open source or commercial products for electronic data capture in clinical trials? A scorecard comparison," Comput. Cardiol., vol. 30, pp. 371-373, 2003

[30] S. J. Rangel, B. Narasimhan, N. Geraghty, and R. L. Moss, "Development of an internet-based protocol to facilitate randomized clinical trials in pediatric surgery," J. Pediatr. Surg., vol. 37, no. 7, pp. 990-994, 2002.

[31] R. Marks, H. Bristol, M. Conlon, and C. J. Pepine, "Enhancing clinical trials on the Internet: Lessons from INVEST," Clin. Cardiol., vol. 24, suppl. V, V17-V23, 2001.

[32] R. A. Hollingsworth, C. Hay, and B. Richards, "An Internet implementation of an international clinical study," Stud. Health. Technol. Inf., vol. 68, pp. 528-531, 1999 .

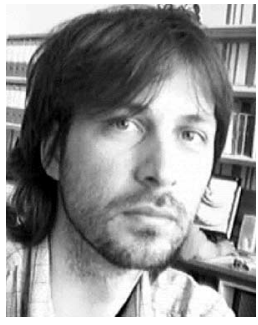

Ivan Pavlović was born in Belgrade, Serbia and Montenegro, in 1973. He received the B.Sc. degree from Belgrade University, Belgrade, in 1999, and the M.S. degree in electrical engineering from the University of Ljubljana, Ljubljana, Slovenia, in 2004.

Currently, he is a Research Associate with the Faculty of Electrical Engineering, University of Ljubljana. He is active in the field of biomedical engineering. His current research interests include Webbased computer systems for clinical trial electronic data collection.

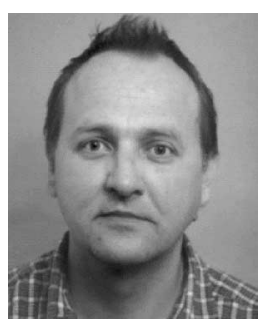

and modeling.
Damijan Miklavčič was born in Ljubljana, Slovenia, in 1963. He received the Ph.D. degree in electrical engineering from the University of Ljubljana, Ljubljana.

Currently, he is a Professor with the Faculty of Electrical Engineering, University of Ljubljana, and the Head of the Laboratory of Biocybernetics. $\mathrm{He}$ is active in the field of biomedical engineering. His current research interests include electroporationassisted drug delivery, including cancer treatment by means of electrochemotherapy, tissue oxygenation, 